Voix et Images

voixetimages

\title{
Menaud, fils de Perrault ou de Savard?
}

\section{Heinz Weinmann}

Volume 3, numéro 3, avril 1978

Pierre Perrault

URI : https://id.erudit.org/iderudit/200119ar

DOI : https://doi.org/10.7202/200119ar

Aller au sommaire du numéro

\section{Éditeur(s)}

Les Presses de l'Université du Québec

\section{ISSN}

0318-9201 (imprimé)

1705-933X (numérique)

Découvrir la revue

\section{Citer cet article}

Weinmann, H. (1978). Menaud, fils de Perrault ou de Savard? Voix et Images, 3(3), 396-407. https://doi.org/10.7202/200119ar d'utilisation que vous pouvez consulter en ligne.

https://apropos.erudit.org/fr/usagers/politique-dutilisation/ 


\section{Menaud, fils de Perrault ou de Savard?}

\begin{abstract}
C'est le pays où l'air est frais comme une source et l'eau, pure comme l'air. Là croissent les gadelles poilues et les viornes et sur les crans austeres brillent les rubis de la canneberge. Jardins mystérieux! retraites inviolées! où, sur le silence des mousses, sans crainte, le caribou mêne la vie limpide des cimes.
\end{abstract}

Menaud, maître-draveur

\section{Menaud moderne contre Menaud bucolique}

Le feu a couvé longtemps sous les cendres. Le voilà de nouveau ravivé par un "testament politique" incendiaire de la main de $\mathbf{M}^{g r}$ FélixAntoine Savard, publié dans le Devoir le 6 janvier 1978. Les passions se déchaînent: pour, mais surtout contre. Le 28 janvier, également dans le Devoir ${ }^{1}$, Pierre Perrault, dans un beau texte qui fera date, à la fois longue complainte et acte d'accusation impitoyable, répond, porte-parole de tous les fils qui se sont sentis abandonnés et trahis par leur père spirituel. II parle au nom de Menaud: "Mon nom est Menaud, dit-il2". Menaud d'un pied ferme quitte son existence livresque et fantômatique, foule le sol de la réalité et, sans crier gare, se tourne contre son géniteur et lui intente un procès.

Tout en lui reprochant ses erreurs du passé, ce royaume qu'il promettait jadis en Abitibi, ce Menaùd rappelle à son auteur que le Québec de 1978 n'est plus le Québec de 1937. Il s'est modernisé. Fini le symbolisme de la Terre! La drave et les draveurs sont morts. Ingénieurs, "navigateurs" et "électroniciens ${ }^{3}$ " ont pris la place vacante! "Dans ce contexte $\mathrm{M}^{\mathrm{gr}}$. Savard, avouez-le, il n'est pas facile d'être Menaud ${ }^{4}$." Certes. Dans ce contexte moderne est-il encore Menaud, peut-il encore l'être?

En effet, qu'est-ce qu'un Menaud sans sa drave? Un Menaud qui a quitté sa chasse, sa montagne chérie et son rang de Mainsal? Un Menaud installé en ville, "à l'horizon fumant des multinationales" ". Que dire d'un Menaud en blue-jeans, planté derrière un haut-fourneau ou penché sur une chaîne de montage? Que dire même d'un Menaud à l'emploi de l'ONF? Peut-on seulement imaginer un tel Menaud? La folie de Menaud n'est-elle pas justement due en grande partie à cette "modernité" et à ces pratiques qui inexorablement envahissent son monde rural? C'est ce monde rural que le nouveau Menaud citadin, avec un air de supériorité que lui donne sa promotion, qualifie de "bucolique ${ }^{6}$. Loin de la terre, Menaud moderne a sans doute oublié les labeurs et les sueurs de sa vie «bucolique» d'antan. 
Dans une telle perspective, ne faudrait-i! pas louer plutôt le père de Menaud pour sa fidélité indéfectible à l'endroit de son fils? En effet, comme son Menaud; il est resté «bucolique». Et c'est ce dont on lui fait grief aujourd'hui: de ne pas être moderne. Au lieu donc de reprocher à $\mathrm{M}^{\mathrm{gr}}$ Savard d'avoir «abandonné" ses fils "à mi-chemin» (les a-t-il jamais suivis?), pourquoi Menaud Moderne ne s'avoue-t-il pas tout simplement qu'ayant vécu pendant quarante ans loin de la drave, il est aujourd'hui dans l'impossibilité de la sentir comme un rapport, comme son rapport au monde? Ce n'est donc pas le père de Menaud qui a abandonné "ses" fils, c'est le nouveau Menaud qui, à la dérive des temps modernes, s'aperçoit avec stupeur, lors d'un 6 janvier, de la distance qui les sépare, de leur éloignement. Au lieu de s'avouer franchement qu'il s'est éloigné lui-même, il se met en colère contre son père, sous prétexte que ce dernier ne l'a pas suivi. Qui a quitté qui? Qui a trahi qui?

$M^{g r}$ Savard, avec une sorte d'intuition géniale, aurait-il pressenti la hargne et la révolte de ses fils? Est-ce pour cela qu'il n'a pas donné de descendance mâle à Menaud? Quel sacrifice pour un auteur qui chante comme un hymne la continuité de la race! Après la mort de son fils Joson, en qui Menaud a placé toute son espérance, qui devait le venger comme tout bon fils en réalisant des choses qu'il n'a pu accomplir, il ne lui reste que sa fille Marie. Mais Marie aussi, par un sursaut de générosité surhumain, sacrifie le bonheur rassurant du "vivre icitte tranquille", son "idéal de vie enclavée ${ }^{7}$ " à la grande aventure de l'homme, à la conquête perpétuelle du pays. Elle renonce au mariage avec Le Lucon au nom de l'amour pour le pays tout entier. "Vivre icitte tranquille... vivre icitte tranquille, ce serait plaisant, murmura-t-elle... oui! mais... il faut penser à tout le pays, aussi... Alors, si tu as de l'amitié pour moi, tu continueras comme Joson, comme mon père ${ }^{8}$ ! A ce moment, la race des Menaud se meurt...

$\mathrm{M}^{\mathrm{gr}}$ Savard a eu beau laisser mourir la race des Menaud sur les cimetières de l'imaginaire, la réalité, friande d'imaginaire, a ressuscité des Joson et des Menaud. Certes, on peut applaudir de ce que la réalité, tout naturellement, ait prolongé ainsi et régénéré un monde amputé et moribond. Mais on est en droit de se demander si ces fils "naturels" de Menaud, qui se placent en dehors de l'enceinte du livre Menaud, maitre-draveur, peuvent encore prétendre parler au nom de Menaud, sans qu'on les accuse d'imposture ou de port de faux papiers d'identité?

Qu'ils témoignent donc tant qu'ils veulent en leur nom propre et qu'ils cessent, modernes, de se réclamer de Menaud! Ont-ils remarqué, ces Menaud réels, que l'auteur a pris soin de verrouiller les deux sorties possibles du roman - le début et la fin - pour empêcher l'échappée du lecteur sur les champs vagues de la réalité? L'entrée débouche sur un livre: Maria Chapdelaine, la sortie sur la folie circulaire de Menaud. Le lecteur, hébété comme Menaud, reste avec une vengeance inassouvie sur le cœur. Situation inconfortable, inacceptable. Son seul salut: mordre 
dans la réalité qui a échappé à Menaud, essayer de conquérir dans la réalité ce que Menaud a perdu dans l'imaginaire. Il faut donc cesser d'être Menaud. Devenir Pierre Perrault, ce à quoi il ne peut tout à fait se résigner. II veut rester aussi Menaud. "Mon nom est Menaud pour toujours ${ }^{9}$." II veut ménager la chèvre maigre ${ }^{10}$ du Menaud bucolique et les choux gras d'un Menaud moderne. Avec ce résultat étonnant qu'il nous présente un autre Menaud, un Rebelle impénitent. "Mon nom est Menaud. Mon nom est Rebelle ${ }^{11}$. »

Mais voilà, ce Menaud moderne, entend-il encore les voix de Maria Chapdelaine, les voix des ancêtres? Après quarante ans, ces voix ne sontelles pas noyées dans le brouhaha des bruits du monde moderne? Ne sont-elles pas celles-là même que $M^{\text {gr }}$ Savard, un 6 janvier, a vainement essayé de conjurer? $\mathrm{Si}$, à notre tour, nous voulons les réentendre, nous devons nous rapprocher du Menaud "bucolique". Pour les comprendre, nous devons lire et relire Menaud, maitre-draveur, comme Menaud s'est fait lire et relire Maria Chapdelaine par sa fille Marie...

\section{Voix et images du pays}

Ce sont les images de ton pays. Menaud, maitre-draveur

Tout commence en effet par une lecture. Lecture-choc de Maria Chapdelaine. Lecture qui rompt la quiétude anesthésiante du train-train quotidien. Soudainement, des voix familières bien qu'anonymes, venues d'un passé lointain, font irruption dans ce monde rural où le geste du travail journalier tenait lieu de verbe. Des voix reprises en chœur par le pays tout entier, comme un hymne à la vaillance et à la gloire des ancêtres. «lci toutes les choses que nous avons apportées avec nous, notre culte, notre langue, nos vertus et jusqu'à nos faiblesses deviennent des choses sacrées, intangibles et qui devront demeurer jusqu'à la fin ${ }^{12}$. "Mais comme le chant du cygne, le chœur de ces voix tire sa beauté mélancolique du sentiment du “déjà plus». Menaud ne sent-il pas que ces voix, en même temps qu'elles la glorifient, sonnent le glas d'une époque à jamais révolue?

En effet, l'harmonie des voix familères du sang est traversée périodiquement de stridences, de sons barbares, à jamais incompréhensibles à Menaud: le bruit d'écus sonnants et trébuchants. "Autour de nous des étrangers sont venus, qu'il nous plaît d'appeler des barbares! ils ont pris presque tout le pouvoir; ils ont acquis presque tout l'argent ${ }^{13}$." Bruits qui ont relayé l'écho des canons des plaines d'Abraham et qui, au début, pouvaient se perdre dans les profondeurs des forêts et dans l'immensité du Territoire. Bruits que le coureur de bois et l'habitant «ignoraient" et refoulaient systématiquement depuis plus de 150 ans et qui, soudainement, se transforment en sommation et en ordres. On dirait même, dans une dernière tentative de refoulement qu'on essaie de les arrêter au seuil du roman, pour les réduire à une existence liminaire, littéraire, à un exergue... 
Voix donc - celles des ancêtres et des étrangers - qui ne sont jamais vraiment intégrées dans la vie quotidienne actuelle comme une praxis de cette réalité. Écoute religieuse, répétition obsessionnelle ou refoulement inconscient, ces voix ne s'articulent jamais en un discours sur ou en un dialogue avec la réalité. En dehors des citations livresques de Maria Chapdelaine, seuls les gestes quotidiens et rituels du «langage silencieux" du corps se font l'écho fidèle des voix et images du passé: lors du travail ou lors d'une gigue.

Personne ne parlait plus maintenant.

La danse allait, légère, sur la pointe des pieds, comme pour un envol, et vêtue de feu.

Délivrés, inlassables, ils exprimaient, chacun, sa vie propre. Ils révélaient par les bras, les pieds, les yeux, les cris poussés dans la frénésie des cadences, ce qu'ils avaient reçu du passé et appris par eux-mêmes; ils animaient d'une sorte de lyrisme sauvage tout ce décor de misère ${ }^{14}$.

La voix de Menaud défaille même lorsqu'il veut répéter, articuler les paroles fatidiques de Maria Chapdelaine. "Des étrangers sont venus, dit-il, ils ont pris presque tout le pouvoir; ils ont acquis presque tout l'argent... "Sa voix sa brisa dans sa gorge ${ }^{15}$. Menaud n'aura d'autre recours que de se faire l'écho, dans son corps et dans son esprit, de l'aliénation qu'il subit dans la vie réelle! son esprit s'aliène.

\section{Entre la fiction et la réalité}

Même si le point de départ de Menaud et de Pierre Perrault est identique, leurs stratégies ultérieures sont à l'opposé l'une de l'autre. Pour les deux, au commencement, une lecture: lecture qui sème le désarroi, l'inquiétude, la révolte. Face à cette lecture, le problème de Menaud pourrait s'énoncer ainsi: comment insérer ce message «politique»de Maria Chapdelaine ("Autour de nous des étrangers sont venus...») dans la réalité " bucolique " quotidienne, comment rendre le texte de Maria Chapdelaine lisible dans son contexte actuel? Le problème de Pierre Perrault: comment insérer la réalité politique dans le cadre «bucolique» de Menaud, maître-draveur?

Concédons à Menaud qu'il a la tâche la plus ingrate, la mission impossible pour un draveur de transformer la réalité pour la rendre conforme à sa «fiction», à l'idéal de Maria Chapdelaine. Travail auquel il n'est pas préparé, travail de politicien. Et il échoue dans cette aépreuve de la réalité" (Freud).

Si de cette “diable de lecture" sont “sortis des mots en armes ${ }^{16}$ ", le tranchant de ces mots s'émousse vite dans l'immensité du cadre naturel. Qui ne saisit le symbolisme de la porte ouverte après la lecture de Maria Chapdelaine? En ouvrant tout grand la porte de la maison, Menaud laisse 
s'échapper ver̀s de vastes horizons, la colère et la rancune accumulée dans son foyer clos. "Puis, il ouvrit la porte toute grande; et, dans le soir immobile, il contempla longtemps la campagne endormie, laissant ses regards voler jusqu'aux horizons lointains ${ }^{17}$.

Menaud interprète et exécute donc à sa façon les ordres reçus de Maria Chapdelaine ( Autour de nous des étrangers sont venus... ») dans les "explōsions de vie» du printemps qui libèrent le pays du joug de l'hiver, lui tiennent lieu de coups de fusil qui pourraient libérer le pays du joug de l'envahisseur. "ll se tenait là, fixé sur ces mots (ceux de Maria Chapdelaine) d'où jaillissaient une force, une jeunesse, quelque chose de comparable au printemps miraculeux de Mainsal avec ses explosions de vie après le froid, la neige, les six longs mois d'hiver ${ }^{18}$."

Une fois en contact avec la Nature, Menaud retrouve sa liberté que la lecture de Maria Chapdelaine lui avait fait croire presque irrémédiablement perdue. "II se sentait libre enfin, humant l'air vif, et jouissant de revoir cette longue bande de forêt riveraine [...] et sous le manteau d'apparence immobile, toute une vie réduite par l'hiver et qui se libérait soudain, se dilatait à l'aurore et s'exaltait en un vol aussitôt replongé dans la forêt humide du matin ${ }^{19}$."

Comme les paroles, les "songes héroïques" (on “jongle" beaucoup dans Menaud) ne se réalisent pas, mais se perdent eux aussi dans les profondeurs des forêts. "Mais comme l'aube était prochaine, le songe héroïque s'enfuit au cœur profond de la forêt vierge où habitent les songes ${ }^{20}$. $)$

Et si les choses en viennent au pire, si trop d'obstacles (embâcles) entravent le cours "normal", naturel des choses - l'ordre des choses une charge de dynamite bien placée, en pleine nature, libérera... des billes qui se disloquaient et se bousculaient dans la Noire." Et ce spectacle lui soulageait le cœur qu'il avait gros depuis la veille, à cause des paroles flétrissantes de Menaud: “Nous sommes des lâches! des lâches ${ }^{21}$ !" On a ou sa revanche, on se sent moins lâche.

Ironie tragique, lorsque l'ennemi le cerne de partout, Menaud fourbit ses armes, en l'occasion ses raquettes, en pensant aux victoires passées et futures sur les champs de bataille de... l'hiver. "ll célèbre, en fredonnant, ce lacis de nerfs et de force avec lequel ses pères ont battu les neiges qui, depuis trois cents ans sont tombées du ciel, ce signe de leur victoire sur les champs rigoureux des hivers infinis ${ }^{22}$."

La Nature, grand exutoire des agressivités de Menaud, désamorce jusqu'à toute velléité 'de combat.

Ce manque de combativité de Menaud, on peut le regretter, mais on n'est pas en droit pour autant de l'esquiver. Après tout, Menaud est un coureur de bois et non un guérillero. On peut regretter également, chez Menaud, une conscience politique, tellement mince qu'elle est 
quasiment inexistante. A ceux qui font des lectures politiques de Menaud, prière d'insérer ce passage:

- Apparence, dit l'un, qu'on va perdre les Hauts. On a beau dire, c'est choquant; tout s'en va aux mains des étrangers.

- Les autres répétèrent:

- T'as raison; c'est choquant; tout s'en va.

- Faudrait tout de même...

Un politicailleur cria:

- Faudrait-il parler de cela au député...

- La farce dérida tout le monde et le peuple se dispersa ${ }^{23}$.

Pour Pierre Perrault, à l'inverse, il s'agit tout simplement, comme pour bien d'autres, d'étirer la peau de chagrin élastique de la fiction de Menaud pour qu'elle épouse les formes rugueuses de sa réalité politique et économique. Nous obtenons alors comme résultat un Menaud qui a certes, beaucoup de panache (le panache de la fiction) et où s'assouvissent - dans une fiction au second degré - des velléités de combats non complètement'satisfaites dans la réalité. Menaud le Rebelle. Menaud le Guérillero, Menaud le héraut d'un Québec indépendant.

De toute façon, à la lecture, tous les «faits" s'ordonnent tellement bien qu'on vérifie même encore rapidement la date de publication de Menaud pour s'assurer si, par hasard, Marx n'aurait pas pu s'en inspirer pour son Capital. «Des étrangers sont venus. " Lisez: les envahisseurs, les capitalistes anglais qui tiennent les leviers du pouvoir et qui spolient le prolétariat canadien-français de ses déjà maigres ressources. La révolte du prolétariat, fomentée par Menaud, échoue, parce que les conditions socio-politiques n'étaient pas encore mûres...

Un seul obstacle: les visées révisionnistes et réactionnaires de l'auteur de Menaud. $\mathrm{M}^{\mathrm{g}}$ Savard bucolique et fédéraliste. "Savard dénie Menaud ${ }^{24}$. Faut-il sous-entendre que Menaud, à l'opposé de $M^{\text {gr }}$ Savard, serait indépendantiste et progressiste? Menaud bâillonné et ligoté par les idéologies hochera toujours la tête: regardez, il a dit oui! Donc pas de problème du côté de la fiction. Reste la réalité: l'auteur de Menaud.

Par un exercice subtil de casuistique, on le coupe en deux: le poète (la fiction), l'homme, le chanoine (la réalité). On loue le poète et son fils spirituel fictif (Menaud) et on flagelle la personne du chanoine, qui, anachronique, insulte ses fils par sa présence trop obsédante. "A Savard le poète je conserve toute mon estime. Il est seulement malheureux qu'un prince de l'église lui ait survécu ${ }^{25}$."

Freud nous a montré que le conscient, pour les brouiller, inverse souvent les messages de l'inconscient. Dans une telle perspective psychanalytique, le titre de l'article de Pierre Perrault «le Royaume des pères à l'encontre des fils " devra se lire en clair: "les fils à l'encontre du royaume des pères". CEdipe, pour devenir Roi lui-même, tue Laïus, son père/rival et se marie ensuite avec Jocaste, son amante/mère. Pierre Perrault tue (symboliquement) son père spirituel pour pouvoir se donner, enfin seul; corps et âme à cette Amante/Mère: le Pays. 


\section{IV. Éros et Thanatos}

Sire, por Dieu omnipotent, II ne m'aime pas, je ne lui, Fors par un herbé dont je bui Et il en but: ce fut pechiez.

Tristan et Iseult

Et redit au passant: qu'elle est morte d'amour, la pauvre Malhurée!

Complainte de la Malhurée

Le Pays, cette "amante affolée", comme on l'appelle dans la "compainte de la Malhurée", à qui les hommes sacrifient tout. On dirait qu'aucun holocauste humain n'est trop grand pour honorer cette déesse virginale implacable: la Nature, la Terre. La Nature, véritable fiancée des hommes, car ils l'aiment jusqu'à la folie, jusqu'à la mort. L'Isolde des Tristans québécois...

Le rapport de Menaud au pays est érotique. II fallait qu'il soit veuf, comme la Malhurée est veuve, pour qu'ils puissent assouvir, en dehors de tout rival gênant, leur passion dévorante. Menaud embrasse la Nature comme on embrasse une amante. "Alors, il avait fait un large geste, comme s'il eut voulu embrasser le pays tout entier ${ }^{26}$." Amour-passion que la Nature lui rend bien. "Cette nature, elle semblait l'aimer depuis le jour, lointain déjà, où il s'était appliqué à la connaître ${ }^{27}$."

L'“ordre des choses", la nature des choses, pour Savard, c'est d'abord cela: la chose de la nature, la passion de la nature. Avant que la politique ne s'en soit mêlée. Qui a pu s'y tromper? Le père lui-même? Peut-être. Mais les fils "naturels" de Menaud, surtout, qui ne sentent plus cette «nature des choses», cette adhésion passionnée à la Nature, des fils qui, au lieu de cela, raisonnent, ergotent, expliquent. Finies les "jongleries" de Menaud! "Il [Savard] m'a forcé de questionner toute la terre pour y trouver une explication ${ }^{28}$."

Car comme Tristan, et c'est cela qui attise sa passion, Menaud n'aime pas la possession, il aime l'Amour. "Ce qu'ils aiment [Tristan et Iseult] c'est l'amour, c'est le fait même d'aimer'29. "Et comme Tristan, Menaud boit son philtre d'amour, "alibi de la passion ${ }^{30}$ ", philtre qui l'arrache aux bras de sa femme, pour le jeter dans ceux de l'Amante/Nature. "Sa femme, avait tout fait pour enraciner au sol ce fier coureur de bois. Et lui, par amour pour elle, il avait défriché cette âpre terre de Mainsal, toujours prêt, cependant, à s'évader du regard vers le bleu des monts dès que le vent du Nord venait lui verser au cœur les paroles magiques et les philtres embaumés ${ }^{31}$."

Le pays qu'aime ainsi Menaud ne s'arrête pas aux frontières territoriales du Québec actuel. C'est le pays des ancêtres qui s'étendait de la 
Baie d'Hudson jusqu'en Louisiane. C'est l'amour d'un Continent, l'amour des horizons infinis, l'amour de l'illimité «lls [les coureurs de bois] s'étaient fait une âme semblable à l'âme des bois, farouche, jalouse, éprise de liberté; ils s'étaient taillé un amour à la mesure des grands espaces ${ }^{32}$."

Car les coureurs de bois, derniers avatars des premiers conquérants, sillonnent inlassablement et presque religieusement (ils ont une mission!) le Territoire, pour lui déclarer, au passage, leur amour. Tâche démentielle, sans limite spatiale, sans fin temporelle. Lorsque l'amour peut ainsi se passer de possession, lorsqu'il se fait quête lointaine, à jamais inachevée, alors Thanatos subrepticement s'en mêle et s'affuble du masque de l'Amour.

Comment ne pas voir jusque dans les films de Pierre Perrault ce lien étroit d'Éros et de Thanatos? Amour du pays, mais aussi amour/haine (fascination) de tout ce qui est déchéance, fin d'un règne. Mort... L'Acadie, l'Abitibi et maintenant la Bretagne.

L'auteur de Menaud a beau mettre en garde son fils fictif contre cet amour mortel de l'illimité, celui-ci n'écoute que sa passion. "Posséder! s'agrandir! Pour ma race, tout autre instinct était un instinct de mort ${ }^{33}$."

Et démesure des démesures, le coureur de bois ne revendique pas seulement les territoires qu'il a parcourus physiquement ou même ceux qu'il pourrait encore parcourir: tout le Territoire, tout un Continent le sollicitent, l'appellent dans leur virtualité virginale, vibrante. Quoi qu'on ait pu dire, le rapport à la terre (à l'image de celui de son amour) n'est pas celui d'un possédant, mais celui d'un appelé qui veille sur la totalité continentale:

Tout le domaine que ses ancêtres avaient conquis, tout alors passa devant les yeux de Menaud, même les lieux, là-bas, au loin, où il n'était jamais allé, où il n'irait sans doute jamais, mais dont il s'est fait en lui une idée vibrante; chaude, une image où l'immense pays avait ses sourires, ses voix, ses chants d'amour et ses appels de détresse, tout comme un être de chair et de sang ${ }^{34}$.

Les catégories possédant/possédé défaillent en Menaud: le plus riche et le plus pauvre, il possède tout et rien. Le problème de Menaud, fondamentalement, n'en est pas un de possédant/possédé, mais un d'ouverture et de clôture, d'accessibilité et de barrières. Certes, on pourrait bien dire aussi qu'il perd l'accès à sa «montagne sacrée » parce qu'il ne s'est jamais préoccupé de la prise de possession de ce territoire. Mais il est justement Menaud parce qu'il ne s'est jamais préoccupé de prise de possession. "Il aurait bien pu se fixer comme les autres, là-bas, qui vivaient à gratter la terre entre les roches, à boulanger des mottes en dedans de leurs clôtures; ceux-là mouraient dans leurs lits ${ }^{35}$. "

Comme il les méprise, ces sédentaires avec leur terre limitée, zonée, clôturée. On y lit «propriété privée, défense d'entrer». Imaginez les rages de Menaud! II enrage de voir de petits esprits se barricader 
égoïstement derrière la clôture de leur terrain, qui leur cache et leur fait oublier jusqu'à l'existence du Territoire immense. Le rêve de l'empire virtuel de Menaud se meurt derrière la clôture de la basse-cour de l'habitant.

Être libre cela ne se bornait pas à dire: “Je fais à ma tête chez moi. Je suis roi et maître de quelques arpents de terre sous le soleil du bon Dieu."

Non! Être libre, c'était, partout où l'on va dans le pays, sentir sous ses pieds, le son de la terre répondre aux battements de son cœur, c'était entendre partout la voix du sol, des bois, des eaux dire: "J'appartiens à ta race et je t'attends!"

\section{L'argent c'est l'ennemi}

Et qui ne voit pas que la haine de Menaud s'exprime d'abord contre les siens, parce que tout simplement les étrangers sont absents de ce roman? On en parle, on ne les voit jamais. Menaud moderne a dû les voir avec les yeux de Pierre Perrault lorsqu'il affirme «il m'a fait apercevoir l'envahisseur, le possesseur ${ }^{37}$ ".

«Tas de lâches! disait-il, qui, dans le péril commun, n'ont pas de ccour au-delà leurs clôtures. " Les propriétaires sédentaires, devenus esclaves de leurs biens, défendent leurs petits intérêts individuels et perdent de vue l'intérêt collectif de la totalité du Territoire. Dans ce contexte, la phrase de Marie prend tout son sens: "ll faut penser à tout le pays. "

L'intégrité du Territoire, violée une première fois, par la clôture des propriétaires sédentaires de la même race, le sera de nouveau - et de façon plus grave, puisqu'on touche à la «montagne sacrée» de Menaud - par l'étranger. Finalement, tout ce qui s'oppose à l'intégrité sacrée de la territorialité devient "étranger», ennemi.

La première violation appelle en châtiment la seconde, puisqu'elle introduit un nouveau circuit, inconnu au coureur de bois: celui de l'argent en tant que pouvoir. L'argent, comme ces "étrangers", circule incognito, rôde, imprévisible comme ce traître Délié (il délie les liens de solidarité du groupe). Assis on fait «travailler», circuler l'argent. C'est l'Argent qui conquiert le pays une seconde fois. II singe parodiquement la prétention à la totalité du coureur de bois. Avec cette différence qu'il ne laisse pas le territoire dans sa virtualité virginale: L'argent veut posséder, tout posséder, comme Délié veut posséder Marie.

«Le Délié est parti d'avant-hier avec des étrangers qui veulent tout avoir. Avec de l'argent, ils l'auront bien. Lui, il aura la garde... mais nous autres... ${ }^{38}$ L'Argent, cet ennemi invisible pour Menaud, n'est visible qu'à l'argent. Seul l'Argent (Pouvoir) combat l'Argent. De façon significative, Menaud vit, imagine sa lutte contre cet ennemi en termes de clôtures qui entravent sa libre circulation sur le territoire. II part combattre cet 
ennemi comme on irait à la chasse à l'outarde. "ll s'était fait une fête de partir ${ }^{39}$." Mais il ne trouvera aucune clôture, aucun ennemi...

\title{
VI. L'homme du ressentiment
}

\begin{abstract}
Le ressentiment est un auto-empoisonnement psychologique, qui a des causes et des effets bien déterminés. C'est une disposition psychologique, d'une certaine permanence, qui, par refoulement systématique, libère certaines émotions et certains sentiments, de soi normaux... et tend à provoquer une déformation plus ou moins permanente du sens des valeurs, comme aussi de la faculté de jugement.
\end{abstract}

Max Scheler, l'Homme du ressentiment.

A défaut d'un ennemi réel, il s'imagine une scène de combat. "ll s'était représenté cent fois la scène: la sommation d'abord, ensuite, sa réponse droite et fière. Puis, la pourchasse de l'intrus jusqu'au bas de la montagne, quelque chose comme une débâcle de toutes les colères que le pays avait sur le cœur depuis les années de servitude. Triomphant, il aurait ensuite regagné ses chasses, les sanctuaires profonds de son domaine, les aires étincelantes de ses lacs; il aurait bu le coup de la liberté à même l'air frais et vierge des monts.",

"Le soir, il se serait enfin reposé en tête à tête avec ses morts consolés ${ }^{40}$." Menaud rêve ce combat comme un duel chevaleresque...

Rien de pire que ces haines, ces rancunes, ces vengeances rentrées, qui ne s'extériorisent plus, qui ne prennent plus pour cible un ennemi extérieur. Ces haines, de façon suicidaire, se tournent soit contre le sujet lui-même, soit contre les siens, contre ceux de sa propre race.

Car Menaud, et c'est ici seulement que le bucolique et le moderne coïncident, est l'homme du ressentiment. D'après Nietzsche qui, le premier, avant Max Scheler, l'a défini, le ressentiment est le propre "de ces êtres à qui la vraie réaction, celle de l'action, est interdite et qui ne trouvent de compensation que dans la vengeance imaginaire ${ }^{41}$. L'homme du ressentiment naît lors d'une blessure, d'un traumatisme que l'Autre, le nonmoi, l'Ennemi lui a infligé. Pour Menaud, c'est la «blessure du patrimoine rétréci et souillé ${ }^{42}$ ", pour le moderne, c'est cette même blessure, avec en plus, la désaffection de ce qu'il croyait être son père spirituel.

Blessure purulente qui ne guérit pas, puisque le sujet, à défaut d'une action réelle, véritable riposte du tac au tac, qui lui donnerait "satisfaction" parce qu'elle lui aurait fait rencontrer son Ennemi (peu importe son triomphe ou sa défaite), à défaut donc de tout cela, rumine 
«jongle» une vengeance future. C'est l'homme qui, ayant refoulé la riposte immédiate et directe à son ennemi, voit des ennemis imaginaires partout. C'est l'homme cerné, traqué par l'Autre. C'est I'homme de l'état de siège permanent. Menaud, dans sa folie va répétant "des étrangers sont venus! Des étrangers sont venus!" Et le moderne de surenchérir, «qu'en est-il aujourd'hui en Abitibi? A Montréal? Sinon l'état de siège. L'investissement. L'envahisseur qui rachète tout: même notre misère ${ }^{43}$ ". A force d'avoir vu l'Ennemi nulle part, il le voit partout...

Le Menaud moderne réussit son coup - c'est-à-dire de rendre invisible son vrai ennemi - par une opération chirurgicale (qu'il reproche d'ailleurs aux autres, qu'il appelle les "chirurgiens du destin», de pratiquer) à cœur ouvert: le sien propre! II "oblitère la conquête" en s'abolissant lui-même comme sujet (historique) agissant et responsable, fût-ce sous l'ironie ducharmienne. "L'autre [défaite], celle de 1760, était la défaite de la France: nous n'y fûmes pour rien: ce fut un marquis qui perdit, disposant, sur un champ, de nos vies et de nos destins selon les règles de l'art ${ }^{44}$." Plus de champs de bataille, plus d'ennemi, plus non plus de sujet responsable à qui il incomberait de se venger de cette humiliation. Humiliation qu'il accueille ensuite comme étant la sienne propre, comme faisant partie de sa condition. De là le ton larmoyant et la complaisance dans le misérabilisme. A l'entendre, on dirait que le Québec est né dans cette épopée héroïco-comique de la "conquête» de l'Abitibi.

Si l'homme $d u$ ressentiment livre ainsi des batailles imaginaires à des ennemis imaginaires, il décharge toute sa hargne, son désir de vengeance contre sa propre personne, contre son semblable, son congénère... son ami (plus faible que son ennemi réel!). Sa devise: «Dieu protégez-moi de mes ennemis, mes amis je m'en charge. " Le prochain, né de la même race, l' "ami" devient ainsi l'ennemi réel. "ll faut d'abord regarder son ennemi pour connaître sa taille et son estoc ${ }^{45}$, nous dit-on. Oui, à condition de le re-connaître.

Ainsi, dans «Menaud», on parle toujours de l'ennemi, mais le seul combat réel auquel on assiste, est le duel entre Délié et Lucon, les deux frères-ennemis du même sang. Ainsi, un 28 janvier, dans un discours qui évoque le spectre d'ennemis innombrables, on exécute publiquement l'ami réel, le vrai, celui qui a transmis à ses fils cette énergie, ce feu dans lequel ils le font périr aujourd'hui. Triste jour aussi que ce 28 janvier.

Peut-être, dans trente ans, Pierre Perrault étant alors aussi octogénaire, par un juste retour des choses, ses propres fils spirituels se dresseront contre lui en lui répétant ce que celui-ci a dit à $\mathrm{M}^{\text {gr }}$ Savard: "Nous nous sommes trompés de combat. "Peut-être alors lui répéteront-ils aussi : «A Pierre Perrault le poète je conserve mon estime. II est seulement malheureux qu'un partisan politique lui ait survécu." Peut-être...

Heinz Weinmann, Collège de Rosemont. 
1. "Le Royaume des pères à l'encontre des fils", le Devoir, 28 janvier 1978.

2. Ibid.

3. Ibid.

4. Ibid.

5. Ibid.

6. Ibid.

7. Ibid.

8. Félix-Antoine Savard, Menaud, maitre-draveur, 1937, p. 146. Toutes nos références renvoient à l'édition Fides, "Collection du nénuphar".

9. "Le Royaume des pères à l'encontre des fils", op. cit.

10. En effet, le Menaud moderne que prétend incarner $P$. Perrault ne veut pas "être réduit à la boîte à lunch, au métier de maître-draveur". Ibid.

11. Ibid.

12. Félix-Antoine Savard, op. cit., p. 12.

13. Ibid.

14. Ibid., p. 31. [Nous soulignons.]

15. Ibid., p. 34.

16. Ibid., p. 15.

17. lbid., p. 13.

18. Ibid. [Nous soulignons.]

19. Ibid., p. 46. [Nous soulignons.]

20. Ibid., p. 14.[Nous soulignons.]

21. Ibid., p. 40.

22. Ibid., p. 111.[Nous soulignons.]

23. Ibid., p. 84-85.

24. "Le Royaume des pères à l'encontre des fils". op. cit.

25. Ibid.

26. Félix-Antoine Savard, op. cit., p. 28.

27. Ibid., p. 50.

28. "Le Royaume des pères à l'encontre des fils ", op. cit.

29. Denis de Rougemont, l'Amour et l'occident, Paris, U.G.E., «10/18», p. 28.

30. Ibid., p. 39.

31. Félix-Antoine Savard, op. cit., p. 14. [Nous soulignons.]

32. Ibid., p. 71.

33. Ibid. [Nous soulignons.]

34. Ibid., p. 103.

35. Ibid., p. 55.

36. Ibid., p. 123.

37. "Le Royaume des pères à l'encontre des fils", op. cit.

38. Félix-Antoine Savard, op. cit., p. 74.

39. Ibid., p. 129.

40. Ibid., p. 130.

41. F. Nietzsche, la Généalogie de la morale, trad. Henri Albert, Paris, Mercure de France, p. 50.

42. Félix-Antoine Savard, op. cit., p. 92.

43. "Le Royaume des pères à l'encontre des fils", op. cit.

44. Ibid.

45. Ibid. 